\title{
Non-invasive identification of polymers in cultural heritage collections: evaluation, optimisation and application of portable FTIR (ATR and external reflectance) spectroscopy to three-dimensional polymer-based objects
}

\author{
Julianne Bell ${ }^{1 *} \mathbb{B}$, Petronella Nel ${ }^{1}$ and Barbara Stuart ${ }^{2}$
}

\begin{abstract}
The conservation of polymer-based cultural heritage is a major concern for collecting institutions internationally. Collections include a range of different polymers, each with its own degradation processes and preservation needs, however, they are frequently unidentified in collection catalogues. Fourier transform infrared (FTIR) spectroscopy is a useful analytical tool for identifying polymers, which is vital for determining storage, exhibition, loan and treatment conditions. Attenuated Total Reflection (ATR), and External Reflection (ER) are proven effective FTIR sampling techniques for polymer identification and are beginning to appear in conservation labs. This paper evaluates and optimises the application of these two FTIR techniques to three-dimensional plastic objects in the museum context. Elements of the FTIR measurement process are investigated for 15 common polymers found in museum collections using both authentic reference sheets, and case study objects to model for surface characteristics. Including: use of the ATR and ER modules, the difference between clamping and manually holding objects in contact with the ATR crystal, use of the Kramers-Kronig Transformation, signal-to-noise ratios for increasing number of co-added scans, resultant time taken to collect each measurement, associated professional, health and safety considerations, and the use and availability of reference materials for polymer identify verification. Utilising this information, a flowchart for applying FTIR spectroscopy to three-dimensional historic plastic objects during museum collection surveys is proposed to guide the conservation profession.
\end{abstract}

Keywords: Polymer identification, Portable ATR-FTIR and ER-FTIR spectroscopy, Three-dimensional plastic objects, Collection surveys

\section{Introduction}

Cultural heritage institutions have significant and growing collections of polymer-based objects which they have a responsibility to preserve for present and future generations. Initially believed to possess long-term physical and chemical stability, a number of plastics have proven to be inherently unstable, also having the potential to

\footnotetext{
*Correspondence: julianne.bell@unimelb.edu.au

1 Grimwade Centre, School of Historical and Philosophical Studies,

University of Melbourne, Parkville, VIC 3010, Australia

Full list of author information is available at the end of the article
}

compromise neighbouring items within a collection. Museum catalogues include minimal polymer identification information, often using general terms such as 'plastic', 'synthetic', 'mixed media' or have no record of plastic materials. However, the identification of plastics is crucial to determining preservation requirements, recording accurate documentation, improving storage environments and choosing treatment pathways. Preliminary data from industry interviews indicates Australian professional conservators are aware of the issues associated with plastics in collections, and the need to identify polymeric materials, however, are lacking access to expertise 
and clear strategies to utilise for collection surveys and identification (unpublished data, Bell 2019).

The majority of literature concerning the identification of polymers in cultural heritage collections is focused on the use of Fourier transform infrared (FTIR) spectroscopy as an accessible, fast and non-invasive technique [1-5]. Additional techniques such as Raman spectroscopy $[5,6]$, near infrared spectroscopy $[5,7]$, gas chromatography-mass spectrometry (GC-MS) $[5,8]$, nuclear magnetic resonance spectroscopy [9], and volatile organic compound (VOC) analysis [10-12] are also utilised. While these techniques have been proven useful, they can require expensive, large and stationary instruments, destructive sampling, and significant time and expertise to operate. Recently, the emergence of portable FTIR instruments include the benefits of a small benchtop footprint, the ability to be moved without the need for recalibration, interchangeable measurement modules and significantly cheaper prices. These features have led to FTIR instruments appearing in gallery, library, archive and museum (GLAM) conservation labs, and has become "the most widely used technique for identifying plastics" [13]. In Australia alone, at least 11 conservation labs have acquired or have direct access to an FTIR spectrometer. Purchase of an instrument also facilitates ongoing application and technical support including in-house training workshops. Additionally, student's undertaking postgraduate studies in conservation are trained in use of FTIR spectroscopy, which has becomes widespread in conservation, and is likely to increase further as an area of expertise in the profession.

Attenuated total reflection (ATR) is the most commonly used FTIR accessory in conservation due to its ability to record high quality infrared spectra nondestructively in less than $1 \mathrm{~min}$. This technique requires the application of pressure between the ATR crystal and the object, ideally with a clamp to ensure intimate contact between the sample and crystal as the IR radiation penetrates to only a depth of $0.3-3$ microns into the sample (dependent on the wavelength, angle of incidence and refractive indices of the reflection element material and sample) [14]. However, plastic collection objects range in shape, size, and fragility, and thus frequently cannot be clamped onto the ATR crystal due to access issues or risk to the object surface. Instead, an IR spectrum can be obtained by applying the pressure manually. Alternatively, an external reflection (ER) module removes the need for contact with the object. Use of these FTIR sampling techniques (ATR and ER) have been established as effective for the identification of polymeric materials in cultural heritage collections [15-17].

Efficacy of these techniques, however, can be limited by the availability of spectral reference libraries and authentic reference materials for identity confirmation. Commercially available ResinKit ${ }^{\mathrm{TM}}$, for example, contains 50 thermoplastics, but lacks $\mathrm{CN}$ and PUR foam, and has also been found to contain incorrectly labelled samples, further complicating identification [18-20]. Spectral libraries available commercially or published do not contain all polymers found in GLAM collections, necessitating reference spectra to be developed in-house. As noted by the POPART round robin testing of the SamCo kit, a comprehensive and high quality reference collection is of crucial importance for plastic materials identification [5]. Best practice guidelines from the pharmaceutical industry include developing in-house reference spectra, multiple identity confirmations, measuring samples on the same instrument and utilising the same parameters [21, 22]. Developed correctly, this process also provides experimental learning with the identification of materials. A collaboration between the Infrared and Raman Users Group (IRUG) [23] and the Bruker Corporation indicates the deficiency in spectral libraries may soon be addressed (personal communication, AIC Meeting 2019). Additionally, in the context of museum collection surveys, measurement procedures need to be clear, concise and reproducible to ensure they can be easily followed by professionals and researchers and applied effectively and consistently to polymeric materials.

An Australian collaboration between three universities, four museums and an art gallery is gathering polymer identification data from ongoing collection surveys. Collating and comparing IR data across partners requires consistent data collection procedures. For this paper, the focus was to evaluate and optimise application of these two FTIR sampling techniques (ATR and ER) to three-dimensional plastic objects in the museum context by determining signal-to-noise ratios (SNRs): first for flat polymer reference sheets, and then compared with case study objects representing various surface and material characteristics. SNRs depend on material characteristics, including refractive index, reflectivity, surface texture, absorptivity and the angle of incidence, which vary significantly between polymers, but can be optimised by the most suitable FTIR sampling technique $[24,25]$. While SNRs are a simplified evaluation measure that does not address all aspects of spectral quality, it is a useful tool for evaluating IR measurements. Utilising this information, a flowchart for applying FTIR spectroscopy to identify three-dimensional historic plastic objects during museum collection surveys is proposed to guide the conservation profession. 
Table 1 Authentic Polymer Reference Sheet Details

\begin{tabular}{|c|c|c|c|c|c|c|c|}
\hline Group & Polymer & Colour & Texture & Sheen & Transparency & Hardness & Source \\
\hline \multirow[t]{2}{*}{ Cellulose esters } & CA & Tortoiseshell & Smooth & Matte & Translucent & Rigid & GPS Agencies, London \\
\hline & $\mathrm{CN}$ & Imitation ivory & Smooth & Gloss & Opaque & Rigid & EBay seller, China \\
\hline \multirow[t]{3}{*}{ Formaldehyde resins } & PF & Orange & Smooth & Gloss & Opaque & Rigid & EBay seller, China \\
\hline & CSF & Cream & Smooth & Matte & Opaque & Rigid & GPS Agencies, London \\
\hline & MF & White & Smooth & Gloss & Opaque & Rigid & EBay seller, Melbourne \\
\hline \multirow[t]{2}{*}{ Styrenics } & PS & White & Smooth & Gloss & Opaque & Rigid & Profile Plastics, Melbourne \\
\hline & $\mathrm{ABS}$ & Black & Smooth & Gloss & Opaque & Rigid & Plastics for Industry, Melbourne \\
\hline \multirow[t]{2}{*}{ Polyolefins } & PP & Grey & Smooth & Gloss & Opaque & Rigid & Plastics for Industry, Melbourne \\
\hline & $\mathrm{PE}$ & White & Smooth & Gloss & Opaque & Rigid & Plastics for Industry, Melbourne \\
\hline \multirow[t]{6}{*}{ Others } & PMMA & Clear & Smooth & Gloss & Transparent & Rigid & Plastics for Industry, Melbourne \\
\hline & PET & Clear & Smooth & Gloss & Transparent & Rigid & Profile Plastics, Melbourne \\
\hline & PC & Clear & Smooth & Gloss & Transparent & Rigid & Plastics for Industry, Melbourne \\
\hline & PVC & Clear & Smooth & Gloss & Transparent & Flexible & Bunnings Warehouse, Melbourne \\
\hline & PUR & Cream & Foam & Matte & Opaque & Flexible & Clark Rubber, Melbourne \\
\hline & PA & White & Smooth & Gloss & Opaque & Rigid & Plastics for Industry, Melbourne \\
\hline
\end{tabular}

\section{Experimental Materials}

Fifteen polymers were selected based on their frequent appearance during a preliminary survey of the Museums Victoria collection [26]. Flat, homogenous polymer reference sheets were purchased from a variety of commercial sources (Table 1) to investigate the impact of polymer type, scan number and FTIR sampling technique on SNR.

Case study objects (Fig. 1) were sourced from antique stores, donations and personal collections to reflect objects surveyed. An effort was made to include a range of unique object characteristics such as shape, colour, transparency, rigidity, sheen and surface texture (Table 2).

\section{FTIR measurements}

Spectra of objects and polymer reference sheets were collected using a Bruker Alpha-P FTIR spectrometer with a diamond ATR crystal or ER sampling modules (Bruker Optik GmbH, Ettlingen, Germany) and OPUS v7.5 software. All spectra were recorded in the range of 4000-375 $\mathrm{cm}^{-1}$, with a spectral resolution of $4 \mathrm{~cm}^{-1}$ in absorbance mode with the ATR module, and reflectance mode with the ER module. A signal check determined infrared radiation was -3199 for ATR and $-10,097$ for ER. Measurements were taken with $8,32,64$ and 128 co-added scans for each sampling technique to investigate the effect of the scan number on SNR. An equivalent number of background scans were conducted before each measurement.

Reference sample sheets were measured by being (1) clamped onto the ATR crystal, and (2) placed in front of the large $\left(433.94 \mathrm{~mm}^{2}\right)$ ER aperture. Case study objects were measured by being (1) clamped, (2) handheld onto the ATR crystal, and (3) placed in front of the small ER aperture with a spot diameter of $3 \mathrm{~mm}$ to accommodate curved surfaces. Before each measurement, the ATR crystal was cleaned using isopropanol and lint free Kimwipes $^{\mathrm{TM}}$ and a cleanness test conducted, where a spectrum is compared with a clean reference spectrum to determine whether the crystal is clean. For materials that are transparent or translucent (PC, PMMA, PVC, PET, $\mathrm{CA}$ ), an aluminium covered microscope slide was placed behind the object in order to reflect the signal back through the sample to the ER module, termed transflectance [14, 27]. In materials conservation, transmission sampling techniques are not used as sample preparation requires sample removal, as a result, transmission databases are not purchased. Instead the profession uses

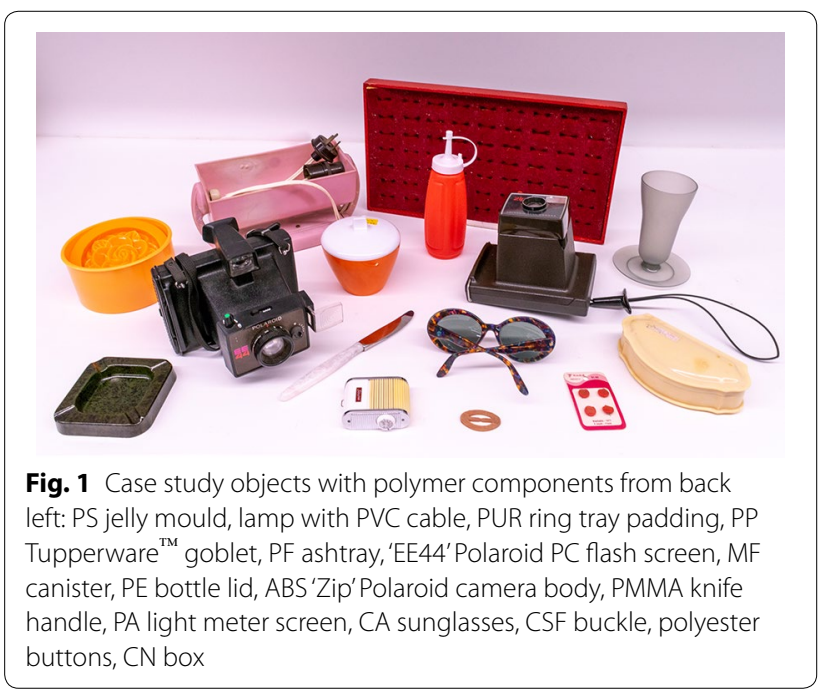


Table 2 Case study object characteristics

\begin{tabular}{|c|c|c|c|c|c|c|c|}
\hline Polymer & Object & Colour & Texture & Sheen & Transparency & Contact shape & Hardness \\
\hline CA & Sunglasses (1990s) & Coloured tortoiseshell & Smooth & Semi-gloss & Translucent & Flat & Slightly flexible \\
\hline $\mathrm{CN}$ & Vanity Box (1940s) & Imitation ivory & Smooth & Gloss & Opaque & Flat & Rigid \\
\hline PF & Ashtray (1940s) & Dark green & Smooth & Gloss & Opaque & Flat & Rigid \\
\hline CSF & Buckle (1950s) & Peach & Smooth & Gloss & Opaque & Flat & Rigid \\
\hline MF & Canister Lid (1955) & White & Smooth & Gloss & Opaque & Curved & Rigid \\
\hline PS & Jelly Mould (1970s) & Orange & Smooth & Gloss & Opaque & Flat & Rigid \\
\hline ABS & Camera Body (1971-5) & Black & Textured & Matte & Opaque & Flat & Rigid \\
\hline PP & Goblet (1980s) & Grey & Textured & Matte & Translucent & Curved & Flexible \\
\hline PE & Bottle Lid (modern) & White & Smooth & Gloss & Opaque & Curved & Flexible \\
\hline PMMA & Knife Handle (1980s) & White pearlescent & Smooth & Gloss & Opaque & Flat & Rigid \\
\hline Polyester & Buttons (1970s) & Orange & Smooth & Matte & Opaque & Flat & Rigid \\
\hline$P C$ & Flash Screen (1978) & Clear & Smooth & Gloss & Transparent & Flat & Slightly flexible \\
\hline PVC & Cable (1950s) & White & Smooth & Matte & Opaque & Curved & Flexible \\
\hline PUR & Padding (unknown) & Cream & Foam & Matte & Opaque & Flat & Flexible \\
\hline PA & Light Meter Screen (1955) & Cream & Textured & Gloss & Opaque & Curved & Rigid \\
\hline
\end{tabular}

Table 3 Plastics conservation workshop samples

\begin{tabular}{|c|c|}
\hline Workshop & Polymer samples \\
\hline $\begin{array}{l}\text { Thea van Oosten 2005, Working with Plastics workshop, 25-26 August 2005, } \\
\text { Museums Victoria }\end{array}$ & PP, PA, PS, PET, CN, CA, PF, CSF, UF, MF, PC, PVC, PMMA, Epoxy, PUR, SBR, GPR \\
\hline $\begin{array}{l}\text { Yvonne Shashoua 2015, AlCCM Conservation of Plastics workshop, 21-24 } \\
\text { July 2015, Museums Victoria }\end{array}$ & $\begin{array}{l}\text { PUR, SI, ABS, PC, plasticised PVC, unplasticised PVC, NR, PE, SBR, CSF, PP, PS, } \\
\text { CN, CA, PMMA, PA }\end{array}$ \\
\hline
\end{tabular}

ATR sampling modules and databases for identification. As a consequence, ATR corrections are not performed on ATR spectra in this investigation, however, if there is access to transmission databases, it would be a worthwhile exercise to perform ATR corrections. Reflectance spectra were also converted to absorbance using the inbuilt OPUS Kramers-Kronig Transformation (KKT), which calculates refractive and absorption indices from the specular data [28].

\section{FTIR analysis: signal-to-noise ratio determination}

An inbuilt OPUS function was used to establish the SNR of all spectra, calculated as peak-to-peak (P-P), across the chosen wavelength window as per industry standards [personal comms. Bruker]. As described [29, 30], the frequency range was set to the standard mid IR (1900-2100 $\mathrm{cm}^{-1}$ ) range to accommodate where the single beam signal intensity is greatest and where minimal IR peaks register.

\section{FTIR analysis: polymer identity verification}

Object spectra were matched against a commercial spectral library (Bruker ATR-FTIR Complete Library, containing more than 26,000 reference spectra, acquired in 2018), using the spectrum search evaluation command. Results of the spectra searches show library spectra with obvious similarities to the unknown and provide a hit quality (HQ) value from 0 (i.e. no resemblance at all) to 1000 (i.e. absolute conformity of the library spectrum with the unknown) [31]. The inbuilt OPUS Quick Compare function, a quality control tool which compares unknown spectra to a user-generated directory of reference spectra by calculating the correlation coefficient for the full spectral range based on peak position and intensity, was also used to evaluate and identify the object spectra. ${ }^{1}$ Unknowns were compared against an in-house database containing spectra of the authentic polymer reference sheets (Table 1), reference samples obtained from two plastics conservation workshops (Table 3) and the ResinKit ${ }^{\mathrm{TM}}$ (Woonsocket, USA) [3]. For further verification, comparison was made with IR spectra published in the literature [15, 32] and the IRUG spectral database [23].

\footnotetext{
1 The correlation coefficient $r$ of the two functions $y_{1}(k)$ and $y_{2}(k)$ is calculated as ratio from the covariance and product of the two standard deviations $\sigma_{y 1}$ and $\sigma_{y 2}: r=\frac{\operatorname{Cov}\left(y_{1}(k), y_{2}(k)\right)}{\sigma_{y 1}-\sigma_{22}}$.

The value of $r$ ranges between -1 (inverted spectra) and +1 (identical spectra). This correlation coefficient $r$ will be calculated into a percentage and reflects the similarity of the two spectra. The $-1 \leq \mathrm{r}<0$ range is mapped to $0 \%$, and the $0<\mathrm{r} \leq 1$ range is mapped linearly to $0 \% \ldots 100 \%$.
} 


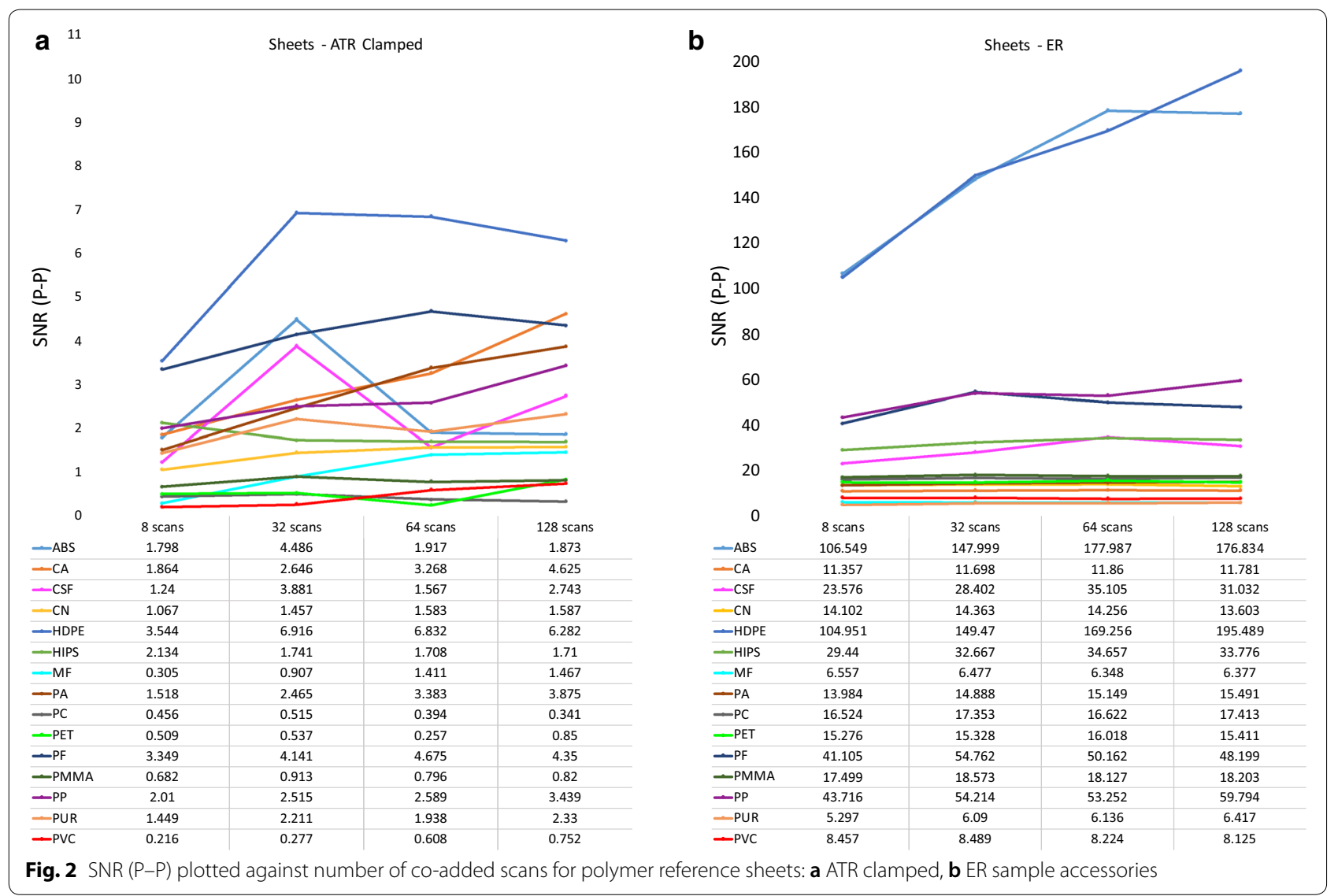

\section{Results}

\section{Signal-to-noise ratios}

SNRs for the polymer reference sheets for the spectra collected using the ATR clamped and ER techniques with 8, 32, 64 and 128 co-added scans are shown in Fig. 2. A comparison of the SNR values demonstrates the ratios are substantially higher for the ER method, approximately of an order of magnitude greater. This is in part due to the threefold increase in IR radiation intensity with the ER module (IR radiation measured at -3199 for ATR and $-10,097$ for ER). With the ATR module, the IR signal is attenuated significantly at the elementsample boundary by the absorption characteristics of the surface layer [14, 33]. Increased co-added scans beyond 32 do not dramatically improve the SNR values for either technique, indicating there may not need to be as much emphasis on a higher number of co-added scans to achieve a good quality spectrum. Observed material characteristics that impact SNR of the polymer reference sheet spectra include transparency, hardness, surface texture, colour and gloss. With the ATR clamped sampling technique (Fig. 2a), transparent materials (PC, PMMA, PET, PVC) produced lower SNR than opaque (PE, PF, PA, PP, CSF, ABS, PS, CN, MF) or semi-opaque materials
(CA, PUR) due to the normal dispersion of light [31]. Hard polymers (MF, PMMA, PC, CF) tend to have lower SNR values, as it becomes more challenging to achieve good optical contact with the diamond ATR crystal [34]. The SNR values produced by the ER module (Fig. 2b) reflect the much higher signal as compared to ATR measurements, particularly for glossy materials (ABS, HDPE), which are highly reflective resulting in a large signal. As with the ATR sampling technique, the same general trend was observed where opaque materials produce higher SNR, and transparent materials lower. Surface texture and gloss impact significantly on SNR with the ER module: highly reflective surfaces (ABS, PE, PP, PF) produce higher SNR, whereas the non-reflective and textured surface of the PUR foam returned a very low SNR.

Measured SNRs for spectra of the case study objects determined for the ATR clamped, ATR handheld, and ER with 8, 32, 64 and 128 scans are plotted in Fig. 3. As with the polymer reference sheets, the SNR values for the ATR clamped measurements are significantly lower than ER measurements due to the amount of signal generated. With all three sampling techniques, the SNRs for the case study object spectra are less consistent than those for the reference sheets, demonstrating 

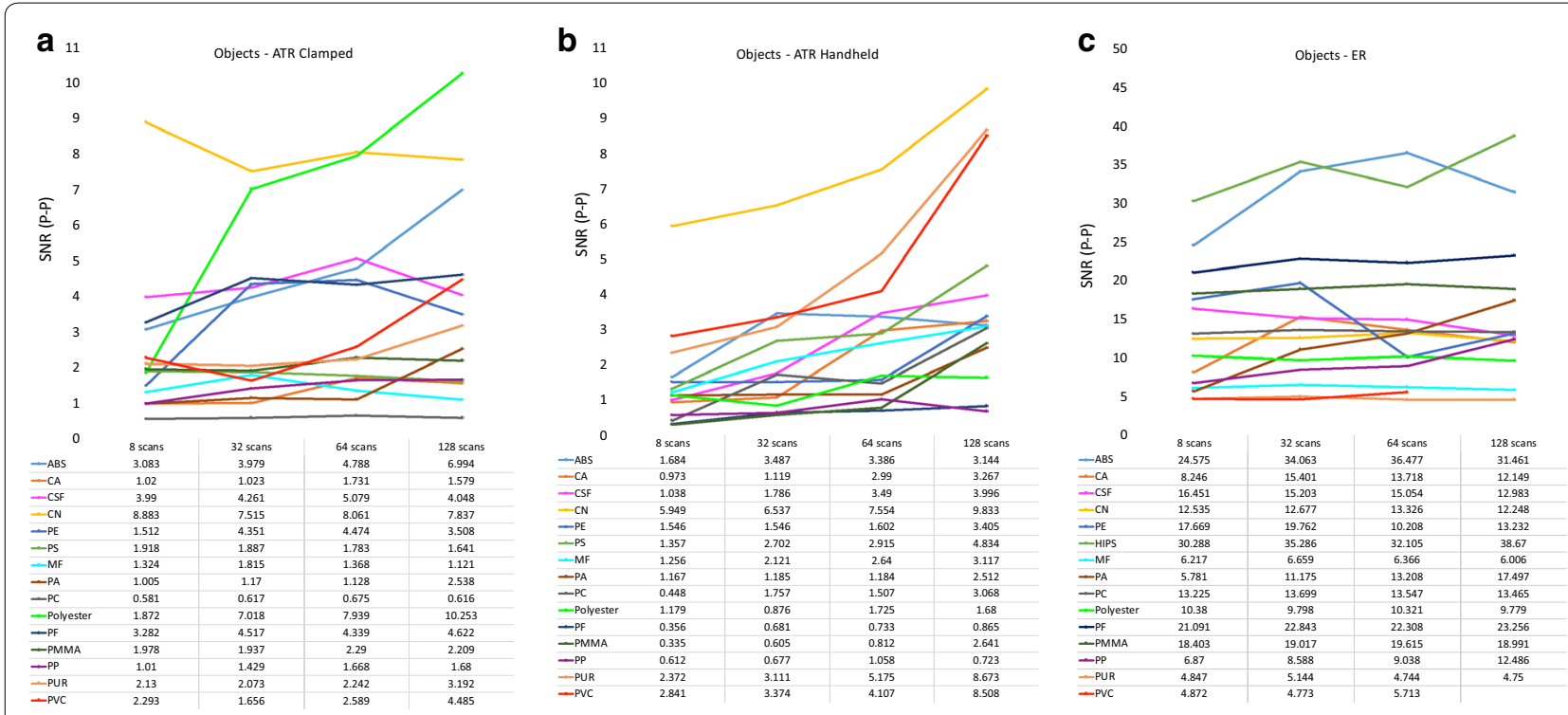

Fig. 3 SNR (P-P) plotted against number of co-added scans for objects: a ATR clamped, b ATR handheld and $\mathbf{c}$ ER sample accessories

the added challenges collection objects present in comparison to flat sheets. The SNRs for the ATR clamped and handheld techniques (Fig. 3a, b) exhibit an increasing trend with more co-added scans, consistent with the multiplex (i.e. Fellgett) advantage [35]. Similar to the polymer reference sheets, SNRs are higher for opaque (ABS, CSF, CN, PE, polyester, PF), and lower for transparent or translucent (CA, PC, PP) objects. The clamping technique showed little difference in SNRs for hard and soft materials, demonstrating its utility.

As previously stated, plastic museum objects frequently cannot be clamped onto the ATR crystal due to their size, shape, fragility or risk to the surface, and thus, require manual pressure to create contact with the ATR crystal. Preliminary experiments demonstrated that during handheld ATR measurements, the operator tends to apply less pressure to the object overtime due to hand and arm strain (data not shown) leading to a drop in SNR as the time taken to conduct measurements increases. In Fig. 3b, however, the SNR values for the spectra generated with the ATR handheld technique are generally consistent across scan number, with improvement with increased co-added scans, demonstrating the operator applied consistent pressure. Close contact is more easily maintained with softer materials (PVC, PUR), and can be challenging with rigid materials (PF, PMMA, Polyester, $\mathrm{MF}$ ), as reflected in SNR values (Figure b). Overall, SNR values are only slightly lower than for the ATR clamped object spectra, indicating manual pressure is an adequate alternative to the clamp when required, however, overall absorbance intensity is reduced.

Examination of Fig. 3c indicates no increase in the SNR with increasing number of co-added scans beyond 32 with the case study objects in ER mode. As for the polymer reference sheets, the ER accessory performs well for opaque, reflective surfaces. The major material characteristics impacting ER measurements are surface texture, gloss and shape. Objects with curved or textured surfaces (PA, PP, PUR, PVC) return lower signals due to the diffuse reflection of the signal in multiple angles now receive by the ER module [27]. Whereas glossy, smooth surfaces (ABS, PS, PMMA, $\mathrm{PF}$ ) produced higher SNR. While the SNR values are high for the ABS and PF objects, their dark colouring (black and dark respectively) impact the spectra resulting in lower overall spectrum intensity with all sampling techniques.

\section{Evaluation of methods for determining polymer identity}

Table 4 summarises the case study object spectral matches for polymer identification, showing the highest accurate HQ against the commercial Bruker ATR-FTIR Complete Library; Quick Compare results against the in-house spectral library obtained from samples (polymer reference sheets, workshop samples, ResinKit ${ }^{\mathrm{TM}}$ ) all measured on the same instrument; and spectral references from literature $(\mathrm{A}=$ Shashoua [32], $\mathrm{B}=$ Saviello et al. [15]) and IRUG database (viewed April 2019). Limitations with each FTIR module and reference source will 
Table 4 Case study object spectral matches for polymer identification

\begin{tabular}{|c|c|c|c|c|c|c|c|}
\hline Object & $\begin{array}{l}\text { Bruker ATR- } \\
\text { FTIR Complete } \\
\text { Library }^{\mathrm{a}} \\
\text { Hit quality } \\
\text { clamped } \\
\text { (handheld) }\end{array}$ & $\begin{array}{l}\text { Reference } \\
\text { Sheets } \\
\text { ATR \% match } \\
\text { clamped } \\
\text { (handheld) }\end{array}$ & $\begin{array}{l}\text { Reference } \\
\text { Sheets }^{\mathbf{b}} \text { ER \% } \\
\text { match }\end{array}$ & $\begin{array}{l}\text { Workshop } \\
\text { Samplesc } \\
\text { ATR \% match } \\
\text { clamped, } \\
\text { (handheld) }\end{array}$ & $\begin{array}{l}\text { ResinKit }^{\mathrm{TM}} \\
\text { ATR \% match } \\
\text { clamped, } \\
\text { (handheld) }\end{array}$ & Literature $^{d}$ & $\begin{array}{l}\text { IRUG database } \\
\text { ATR }\end{array}$ \\
\hline PS Jelly Mould & $875(837)$ & $\begin{array}{l}96.42 \\
(93.72)\end{array}$ & 92.35 & $\begin{array}{l}99.24 \\
(98.19)\end{array}$ & $\begin{array}{l}99.51 \\
(94.25)\end{array}$ & $\begin{array}{l}\text { A: p.266 } \\
\text { B: p. } 18\end{array}$ & ISR00031 ISR00125 \\
\hline PVC Lamp Cable & - & $\begin{array}{l}86.13 \\
(79.97)\end{array}$ & 78.43 & $\begin{array}{l}88.68 \\
-\end{array}$ & $\begin{array}{l}89.43 \\
(85.50)\end{array}$ & $\begin{array}{l}\text { A: p.270 } \\
\text { B: p.19 }\end{array}$ & ISR00155 ISR00063 \\
\hline PUR Padding & 899 (864) & $\begin{array}{l}99.03 \\
(83.10)\end{array}$ & 93.67 & $\begin{array}{l}99.08 \\
(95.54)\end{array}$ & - & $\begin{array}{l}\text { A: p.268 } \\
\text { B: p.19 }\end{array}$ & ISR00157 \\
\hline PP Goblet & $921(847)$ & $\begin{array}{l}98.78 \\
(96.58)\end{array}$ & 71.01 & $\begin{array}{l}99.66 \\
(97.22)\end{array}$ & $\begin{array}{l}98.13 \\
(94.15)\end{array}$ & $\begin{array}{l}\text { A: p. } 265 \\
\text { B: p. } 15\end{array}$ & ISR00058 \\
\hline PF Ashtray & - & $\begin{array}{l}97.24 \\
-\end{array}$ & 78.09 & $\begin{array}{l}95.88 \\
-\end{array}$ & - & $\begin{array}{l}\text { A: p. } 259 \\
\text { B: p. } 10\end{array}$ & - \\
\hline PC Flash Screen & $868(905)$ & $\begin{array}{l}99.39 \\
(92.97)\end{array}$ & 96.25 & $\begin{array}{l}99.73 \\
(99.37)\end{array}$ & $\begin{array}{l}99.73 \\
(94.39)\end{array}$ & $\begin{array}{l}\text { A: p.261 } \\
\text { B: p.11 }\end{array}$ & ISR00122 \\
\hline MF Canister Lid & - & $\begin{array}{l}99.35 \\
(99.04)\end{array}$ & 94.85 & $\begin{array}{l}96.48^{*} \cup F \\
(95.06)\end{array}$ & - & B: p.8 & - \\
\hline PE Bottle Lid & $911(900)$ & $\begin{array}{l}99.22 \\
(96.20)\end{array}$ & 73.85 & $\begin{array}{l}98.15 \\
(97.36)\end{array}$ & $\begin{array}{l}98.51 \\
(96.60)\end{array}$ & $\begin{array}{l}\text { A: p.262 } \\
\text { B: p.13 }\end{array}$ & ISR00077 ISR00135 \\
\hline ABS Camera Body & $770(795)$ & $\begin{array}{l}81.35 \\
(86.63)\end{array}$ & 68.31 & $\begin{array}{l}94.25 \\
(94.88)\end{array}$ & $\begin{array}{l}94.41 \\
(94.89)\end{array}$ & B: p.3 & ISR00095 \\
\hline $\begin{array}{l}\text { PMMA Knife } \\
\text { Handle }\end{array}$ & 909 (866) & $\begin{array}{l}99.22 \\
(95.47)\end{array}$ & 88.49 & $\begin{array}{l}99.27 \\
(98.67)\end{array}$ & $\begin{array}{l}98.84 \\
(98.53)\end{array}$ & $\begin{array}{l}\text { A: p.264 } \\
\text { B: p.5 }\end{array}$ & ISR00080 ISR00081 \\
\hline $\begin{array}{l}\text { PA Light Meter } \\
\text { Screen }\end{array}$ & $884(876)$ & $\begin{array}{l}94.62 \\
(94.65)\end{array}$ & 89.96 & $\begin{array}{l}97.63 \\
(96.65)\end{array}$ & $\begin{array}{l}97.37 \\
(97.82)\end{array}$ & $\begin{array}{l}\text { A: p. } 260 \\
\text { B: p.9 }\end{array}$ & ISR00126 ISR00136 \\
\hline CA Sunglasses & 937 (901) & $\begin{array}{l}98.35 \\
(98.04)\end{array}$ & 74.02 & NA_mislabeled? & $\begin{array}{l}97.06 \\
(93.01)\end{array}$ & $\begin{array}{l}\text { A: p.256 } \\
\text { B: p.6 }\end{array}$ & ISR00154 ISR00064 \\
\hline CSF Buckle & $876(880)$ & $\begin{array}{l}97.56 \\
(97.09)\end{array}$ & 73.25 & $\begin{array}{l}98.55 \\
(98.45)\end{array}$ & - & B: p.5 & - \\
\hline Polyester Buttons & $787(-)$ & $\begin{array}{l}75.88 \\
(49.02)\end{array}$ & 21.27 & $\begin{array}{l}96.05 * \mathrm{GRP} \\
-\end{array}$ & - & $\begin{array}{l}\text { A: p. } 263 \\
\text { B: p.8 }\end{array}$ & ISR00145 ISR00158 \\
\hline CN Vanity Box & $902(814)$ & $\begin{array}{l}98.90 \\
(90.03)\end{array}$ & 92.77 & $\begin{array}{l}99.37 \\
(98.42)\end{array}$ & - & $\begin{array}{l}\text { A: p.257 } \\
\text { B: p.7 }\end{array}$ & ISR00004 ISR00066 \\
\hline $\begin{array}{l}\text { Total positive } \\
\text { identifications: }\end{array}$ & $12 / 15(11 / 15)$ & $\begin{array}{l}15 / 15 \\
(13 / 15)\end{array}$ & $14 / 15$ & $\begin{array}{l}13 / 15 \\
(11 / 15)\end{array}$ & $\begin{array}{l}9 / 15 \\
(9 / 15)\end{array}$ & $\begin{array}{l}A: 12 / 15 \\
B: 15 / 15\end{array}$ & $12 / 15$ \\
\hline
\end{tabular}

be discussed. Peak positions recorded for all spectra are available in Additional file 1.

\section{FTIR module and sampling techniques}

With the ATR module, the spectral absorbance intensity was significantly higher for clamped measurements than for handheld, producing better spectra. However, the SNRs were comparable for the two techniques, and identification was still achievable in most cases, demonstrating the quality of instrument and spectra despite contact technique. Where the ATR handheld technique produced better spectra (ABS, PA objects; Figs. 6c, 8g), it was due to the object's shape impeding performance of the clamp, suggesting that when an object is unable to be easily clamped onto the ATR crystal, manually holding the object is preferable. The handheld technique produced poorer spectra for the PF and Polyester objects (Figs. 5a, 8e) due to the hardness of the materials preventing good contact with the ATR crystal. Spectral features such as the upward baseline shift (more significant at low wavenumbers) in the PF, MF, CSF, CA and PVC sample absorbance spectra are attributable to the infrared radiation scattering from additives, such as fillers or colourants including carbon black and titanium 

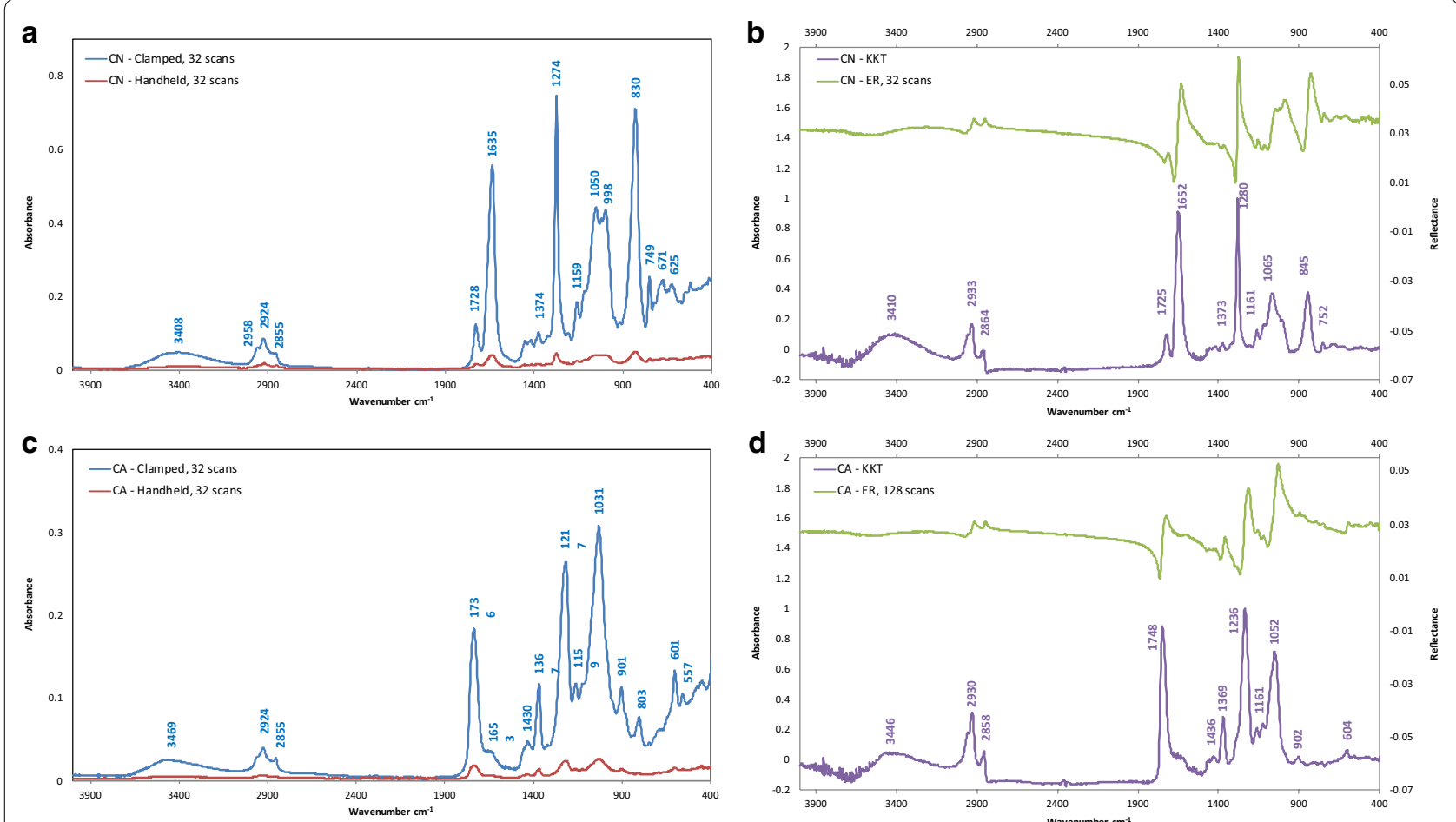

Fig. 4 FTIR spectra for a CN box ATR clamped and handheld, b CN box ER and KKT, c CA sunglasses ATR clamped and handheld, $\mathbf{d}$ CA sunglasses ER and KKT

dioxide. Whereas spectra of the highly transparent plastics (PMMA, PC, PE) show flat baselines [36]. The baseline shift occurs at lower wavenumbers as the depth of penetration of the signal increases, the scattering effect due to the additive also increases [36].

Reflection spectra do not resemble absorption spectra, being determined by the refractive index according to the Fresnel equations [34]. When the KKT is applied to convert the reflectance spectra into absorbance, peak shifts occur, along with band distortions, baseline curves (Polyester), negative baselines and loss of some peaks. In general, if the reflectance spectrum is of poor quality, the KKT of the spectrum into absorbance is also of poor quality (PVC, PUR).

\section{Reference source evaluation}

In ATR mode, only the in-house library of absorbance reference sheet spectra successfully identified all 15 polymers with the clamped sampling technique. Due to not all relevant polymers being including in the IRUG spectral database, Bruker, ResinKit ${ }^{\mathrm{TM}}$, workshop sample and Shashoua [25] spectral libraries, these were unable to identify all case study objects. In ER mode, the in-house library of reflectance reference sheet spectra identified 14 out of 15 (excluding polyester), while Bruker library and IRUG database do not provide reflectance spectra.
The spectral database in Saviello et al. [15], is the most comprehensive resource with 60 polymer spectra in both absorbance and reflectance and matches for all object unknowns.

\section{Cellulose Based: CA, CN}

The $\mathrm{CA}$ and $\mathrm{CN}$ objects were easily identifiable with the ATR clamped and handheld sampling techniques (Fig. 4a, c). In ER mode, the spectrum of the $\mathrm{CN}$ was identified when matched against the reference sample spectrum (Fig. 4b). The CA sunglasses required the coaddition of 128 scans to be identified against the reference sheet with Quick Compare (74.02\%), however, the spectra were visually identifiable against the reference (Fig. 4d).

\section{Formaldehyde resins: $\mathrm{PF}, \mathrm{CSF}, \mathrm{MF}$}

The formaldehyde based resins, PF, MF and CSF were the most problematic to identify due to their hard, rigid structure making it difficult to achieve high quality spectra, and a lack of reference materials for these polymers. When matched against the in-house spectral library of polymer reference sheets, however, all three spectra of the samples are identifiable via ATR clamped and ER (Fig. 5a, c, e). With ATR handheld, the absorbance intensity is low, however, spectral features are still 

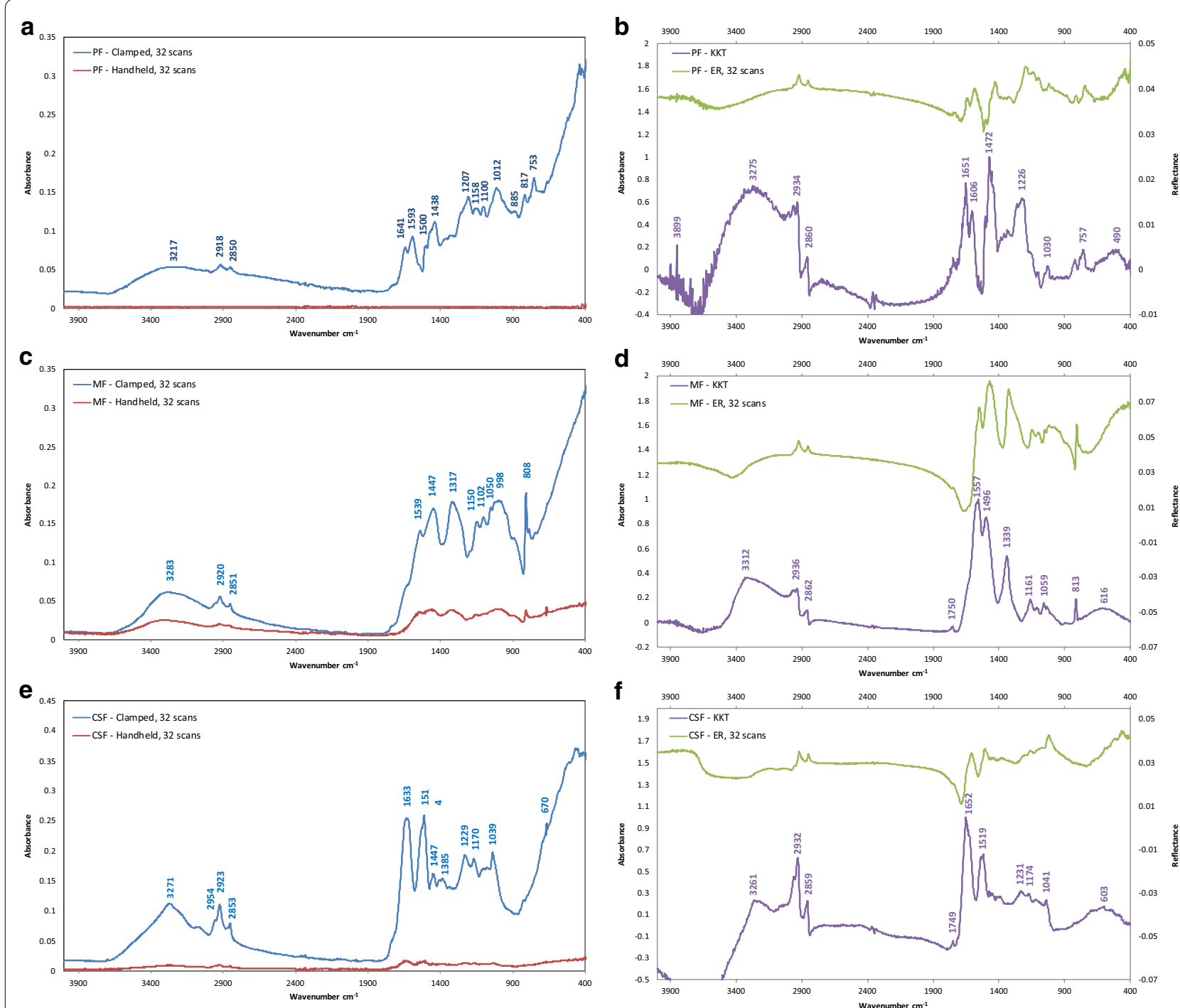

Fig. 5 FTIR spectra for a PF ashtray ATR clamped and handheld, b PF ashtray ER and KKT, c MF canister ATR clamped and handheld, d MF canister ER and KKT, e CSF buckle ATR clamped and handheld, f CSF buckle ER and KKT

discernible for MF and CF (Fig. 5c, e). No spectral features are evident for the PF sample spectrum recorded with ATR handheld (Fig. 5a). PF spectra are of a lower quality overall, attributable to the dark green colouring making it difficult to achieve a good spectrum. Baseline shifts impacted all absorbance spectra, attributable to the infrared radiation scattering from additives, such as fillers or colourants due to higher levels of fillers or other additives (Fig. 5a, c, e). The MF object spectrum collected in ATR clamped mode (Fig. 5c), matched to a Urea Formaldehyde (UF) workshop sample, despite the library containing an MF sample spectrum. The incorrect match indicates an issue with ATR measurements of formaldehyde based resin materials as the resulting spectra are very similar, however, differences in the relative height of the similar peaks allows identification.

\section{Styrenics: PS, ABS}

High quality IR spectra were recorded for all PS measurements due to the highly absorbing nature of the material (Fig. 6a, b). The ABS 'Zip' Polaroid Instant Camera body was more problematic to measure due to its shape, colour and surface texture. As it is a difficult shape to clamp onto the ATR crystal, the handheld measurement produced higher absorbance intensity, a less noisy spectrum and higher identification matches (Fig. 6c). The ABS camera produced poor ER spectra due to the black colour and textured surface, although the polymer is still 

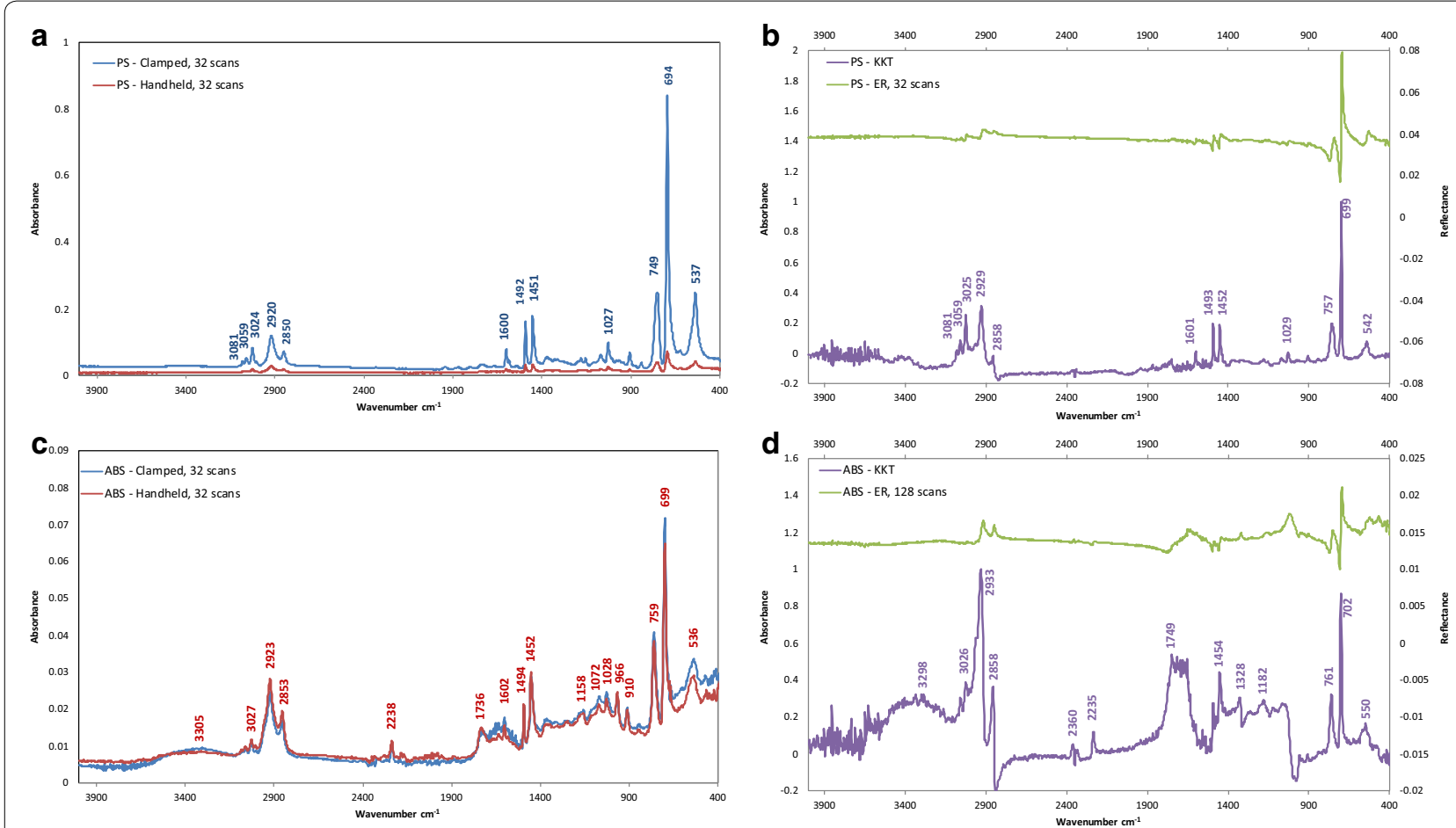

Fig. 6 FTIR spectra for a PS jelly mould ATR clamped and handheld, b PS jelly mould ER and KKT, c ABS camera body ATR clamped and handheld, d ABS camera body ER and KKT

spectroscopically identifiable as ABS when the spectrum is compared to the reference (Fig. 6d).

\section{Polyolefins: PE, PP}

All ATR spectra for the PE and PP reference sheets and objects were of high quality and easily measured with successful identifications (Fig. 7a-d). In ER mode, the PE spectra exhibit noise, possibly due to human error as the bottle lid had to be held in place in front of the aperture. The surface texture and rounded shape of the PP object resulted in poorer quality reflectance spectra. More coadded scans improved the spectrum slightly and a 71.01\% match for identification against the reference sheet was achieved with 128 scans, however, the objects' shape and surface is not well suited to ER measurements.

\section{Others: PC, PMMA, Polyester, PA, PVC, PUR}

High quality spectra were recorded for all measurements of the PC Polaroid camera flash screen and PMMA knife handle with positive identifications (Fig. 8a-d). With the ER module, the PC object was identified successfully utilising transflectance, while there were some minor issues with the PMMA object, most likely due to human error as the knife had to be handheld during measurement.
Issues were identified with spectral matches between the PET reference sheet and polyester buttons due to differences in formulation (Fig. 8e, f). The PET reference sheet is a clear thermoplastic polyester with glycol modification (PETG). Whereas the buttons have a matte, opaque orange surface, and were identified as a thermoset polyester resin through spectrum matches with a glass reinforced polyester (GRP) workshop sample and published Ref. [13]. This highlights the need for multiple, high quality reference materials and spectra for all polymers.

The PA light meter rolling screen, manufactured by Gossen in Erlangen, West Germany, 1955, was particularly difficult to analyse due to its shape, recording a high number of rejected scans with both modules. Despite poor spectra, the polymer was identifiable with the ATR clamped and handheld techniques (Fig. 8g). Reflectance spectra were noisy due to diffuse reflection of the signal from a curved and uneven surface (Fig. 8h). More coadded scans improved spectral quality, with 128 scans providing a positive identification match against the reference sheet spectrum.

The plasticised PVC cable cover produced good absorbance spectra (Fig. 8i), however, spectral comparisons were not as high as for other polymers, which may be attributed to differences in formulation: the 

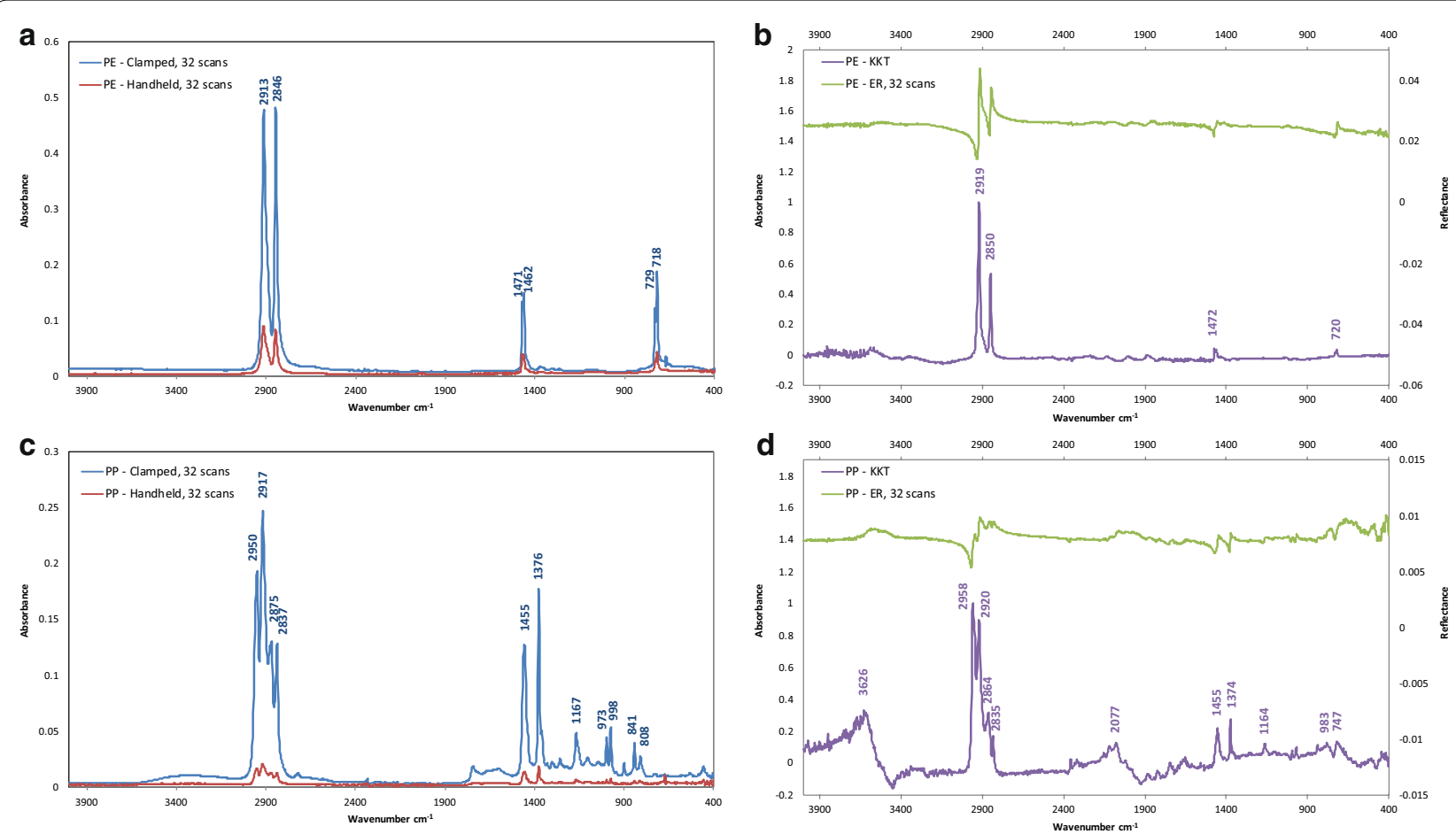

Fig. 7 FTIR spectra for a PE bottle bid ATR clamped and handheld, b PE bottle lid ER and KKT, c PP goblet ATR clamped and handheld, $\mathbf{d}$ PP goblet ER and KKT

reference sheet and samples are new and clear, while the object is aged and opaque white. The object spectrum exhibited strong peaks at $1721(\mathrm{C}=\mathrm{O}$ stretching), 1256 and 1122 (C-O stretch) and small bands at 1600 and $1579 \mathrm{~cm}^{-1}$ (C-C aromatic ring stretching), all indicating the presence of a phthalate plasticiser [37]. After analysing the PVC cable with both ATR techniques, residue remained on the diamond crystal highlighting the necessity of cleaning the crystal. With the ER module, the rounded shape of the PVC cable and minimal surface area impacted analysis and resulting spectrum quality. 128 scans could not be completed due to continuous rejected scans, and the reflectance spectra exhibit signs of saturation [38].

The PUR foam ring tray was identifiable with both the ATR clamped and handheld sampling techniques. The handheld 32 scan spectrum matched to Poly(Vinyl Acetate) in the Bruker library, which after further inspection was to be due to adhesive on the underside of the foam, highlighting the need for accuracy with measurement location. As such, the handheld spectrum displayed in Fig. $8 \mathrm{f}$ is 128 scans. In ER mode, the spectra of both the reference sheet and object were extremely poor due to the foam texture. Many rejected scans were recorded with both and the resulting spectra appear distorted. Spectra were not improved with accumulated scans as the material is not suited to ER analysis. Despite the poor quality, the object was still a 93.67\% match with the sample sheet.

\section{Discussion}

The different instrumental operations of the ATR and ER modules determine their efficacy with the various polymer types and material characteristics. As mentioned the ATR module requires intimate contact between the higher refractive index, infrared transparent internal reflection element (diamond crystal), and sample. Its spectrum is recorded with IR radiation that is incident through the reflection element at an angle greater than the critical angle [34]. The IR radiation is totally internally reflected at the crystal-sample boundary; the infrared beam may, however, be considered to penetrate a short distance into the sample $(0.3-3 \mu \mathrm{m})$ and be attenuated by the absorption characteristics of the sample surface [14]. Alternatively, pure specular reflection spectra can be recorded with the ER module directly from the surfaces of flat, non-scattering (reflective), optically thick (opaque) samples [14]. Diffuse reflection is seen when radiation penetrates below the surface before being scattered or reflected black from within the sample and requires an accessory to collect the IR radiation emerging over a wide range of angles [34]. Most commercial 

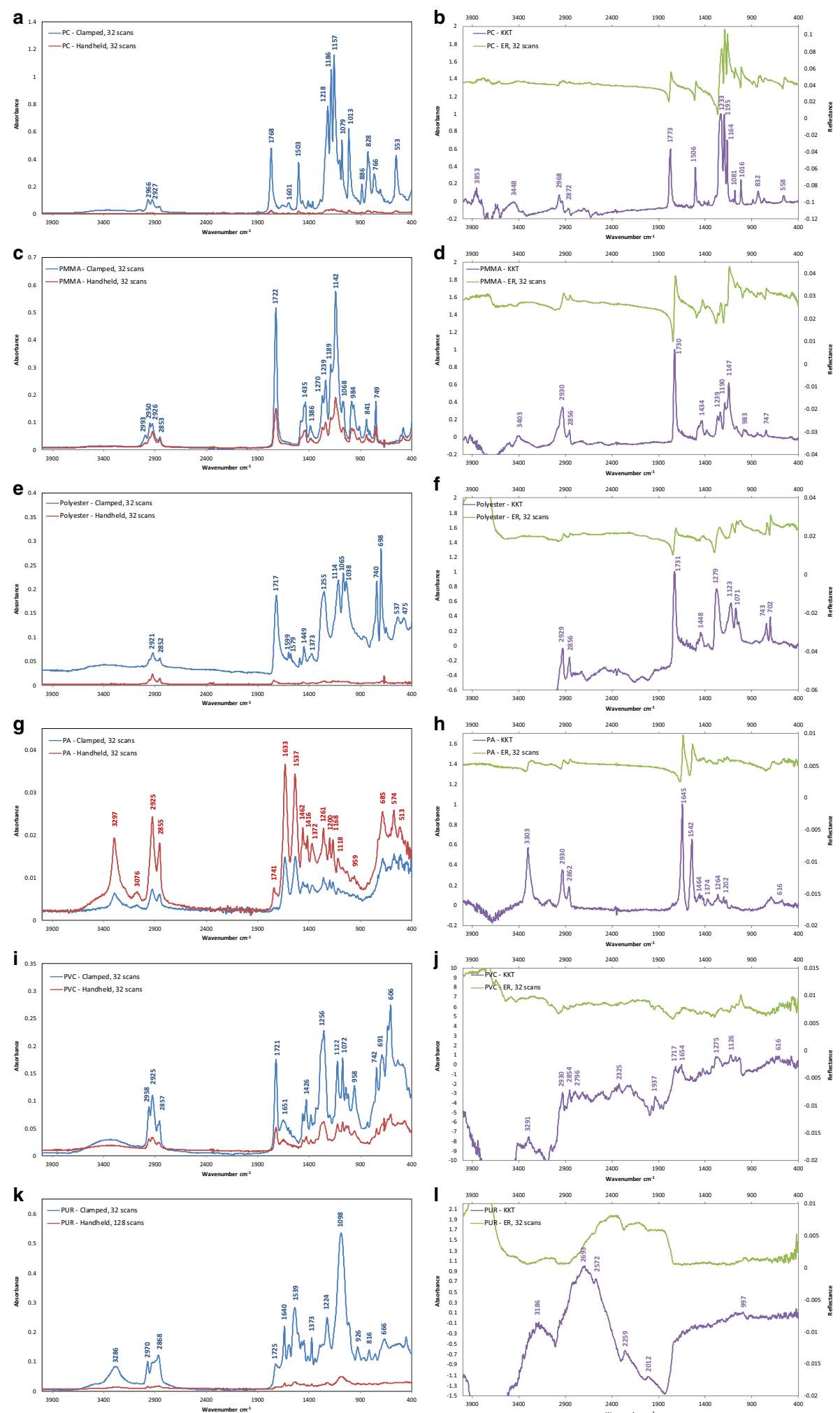

Fig. 8 FTIR spectra for a PC flash screen ATR clamped and handheld, b PC flash screen ER and KKT, c PMMA knife handle ATR clamped and handheld, $\mathbf{d}$ PMMA knife handle ER and KKT, e Polyester buttons ATR clamped and handheld, $\mathbf{f}$ Polyester buttons ER and KKT, $\mathbf{g}$ PA light meter screen ATR clamped and handheld, $\mathbf{h}$ PA light meter screen ER and KKT, i PVC lamp cable ATR clamped and handheld, $\mathbf{j}$ PVC lamp cable ER and KKT, k PUR foam ATR clamped and handheld, I PUR foam ER and KKT 
Table 5 Sampling technique suitability by polymer

\begin{tabular}{|c|c|c|c|c|}
\hline Object & Polymer & ATR clamped & ATR handheld & ER \\
\hline Sunglasses & CA & $\checkmark$ & $\checkmark$ & $\checkmark$ \\
\hline Vanity box & $\mathrm{CN}$ & $\checkmark$ & $\checkmark$ & $\checkmark$ \\
\hline Ashtray & PF & & $x$ & $\checkmark$ \\
\hline Buckle & CSF & & $x$ & $\checkmark$ \\
\hline Canister lid & MF & & $x$ & $\checkmark$ \\
\hline Jelly mould & PS & $\checkmark$ & $\checkmark$ & $\checkmark$ \\
\hline Camera body & ABS & $\boldsymbol{J}^{*}$ & $\checkmark$ & $J^{*}$ \\
\hline Goblet & PP & $\checkmark$ & $\checkmark$ & $\boldsymbol{J}^{*}$ \\
\hline Bottle lid & $P E$ & $\checkmark$ & $\checkmark$ & $\checkmark$ \\
\hline Knife handle & PMMA & $\checkmark$ & $\checkmark$ & $\checkmark$ \\
\hline Buttons & Polyester resin & $\checkmark$ & $\boldsymbol{J}^{*}$ & $\checkmark$ \\
\hline Flash screen & $P C$ & $\checkmark$ & $\checkmark$ & $\checkmark$ \\
\hline Cable & PVC plasticised & $\checkmark$ & $\checkmark$ & $x$ \\
\hline Padding & PUR foam & $\checkmark$ & $\checkmark$ & $x$ \\
\hline $\begin{array}{l}\text { Light meter } \\
\text { screen }\end{array}$ & PA & $\checkmark^{*}$ & $\checkmark$ & $\boldsymbol{J}^{*}$ \\
\hline
\end{tabular}

$\checkmark$ polymer suitable for technique; $\boldsymbol{x}$, polymer not suitable for technique; $\boldsymbol{V}^{*}$, polymer suitable for technique but object characteristics impacted spectra

accessories, including the Bruker Alpha-p ER module, focus the radiation on a small region $(\sim 3 \mathrm{~mm}$ diameter $)$ and collect over a wide but limited range of angles. Thus, the spectra obtained represent a mixture of reflection from the surface and from within the sample [34]. The depth of penetration for reflectance will be different and dependent, in part, on the optical transparency of the material.

The relationship between spectral resolution and SNR is an increase in SNR with decreased resolution, which may be improved by signal accumulation, or an increased number of scans [30]. There is a trade-off, however, between resolution, SNR, and time taken to conduct the increasing number of scans. SNR is one rubric with which to interrogate spectral quality, while not all aspects of the measurement and resulting spectra are assessed, it remains a useful tool for quantitative spectra evaluation. Results indicate that for the ATR module, SNR values over $\sim 0.5$ are required for identification and over $\sim 5$ for the ER module. However, as discussed, the FTIR module, sampling technique, polymer type, effective contact, surface and material characteristics impact SNR more than the number of co-added scans.

Polymer identification with FTIR spectroscopy can be enhanced by utilising the optimum module and sampling technique. Certain polymers and objects are more suited to analysis with either the ATR or ER module due to their material characteristics, summarised in Table 5. Soft, flexible and textured materials, such as the PVC cable cover and the PUR foam ring tray padding are highly suited to ATR analysis as it is easy to achieve close contact with the ATR crystal. However, these are unsuitable for ER, as the textured surface does not reflect sufficient signal to obtain a good reflectance IR spectrum. Conversely, hard, rigid, glossy materials, particularly the formaldehyde resins, can be problematic to measure with the ATR technique as it is difficult to create close optical contact with the diamond crystal. As contact is confined to small areas, weak spectra are produced, the effects of which are greatest at shorter wavelengths where the depth of penetration is lowest [34]. This effect is obvious on the combined CSF, MF, PF sample ATR clamped spectra in Fig. 9a. Such hard polymers, along with carbon-filled polymers, are generally better suited to the ER module that overcomes contact and scattering issues. This produces more differentiation of peaks, assisting in distinguishing between and identifying hard polymers such as PF, CSF and MF (Fig. 9b). Applying KKT to the reflectance spectra, maintains the more distinguishable peaks, and can be useful to analyse spectra and relate them to absorbance data (Fig. 9c). Other highly absorbing polymers such as CN, PS, PP, PE and PC are suitable for all techniques. It is important to note, however, that while a polymer might be suitable for a technique, object characteristics may still impede analysis, as for the round, textured surface of the PP goblet with the ER module, the hardness of the polyester buttons with ATR handheld, and the black colour of the ABS camera.

Clamping objects onto the ATR crystal generally produces a good quality spectrum. Placing a piece of cardboard or blotter paper between the clamp and the object avoids damage to the surface and increases grip strength with smooth surfaces. If clamping is not possible for the object due to shape, size or fragility, consistent handheld pressure provides adequate contact permitting the collection of good quality spectra, particularly with nonrigid materials. When holding the object in contact with the ATR crystal, standing upright over the spectrometer facilitates the application of consistent downward pressure, avoiding fingers or gloves touching the crystal. The conservator operating the instrument (Bell) found it easier to obtain good contact with the crystal via an edge or corner than with a flat area.

When conducting measurements with the ER module, ensuring the object is stationary and flush in front of the aperture has a significant impact on spectral quality, increasing signal strength, minimising noise and reducing rejected scans. Use of the inbuilt camera is a crucial aid ensuring the surface is in focus and usefully documents the measurement location. Best practice is to secure the object with blocks, removing the need to hold anything and the impact of even slight vibrations and shifts due to human movement. For objects with 


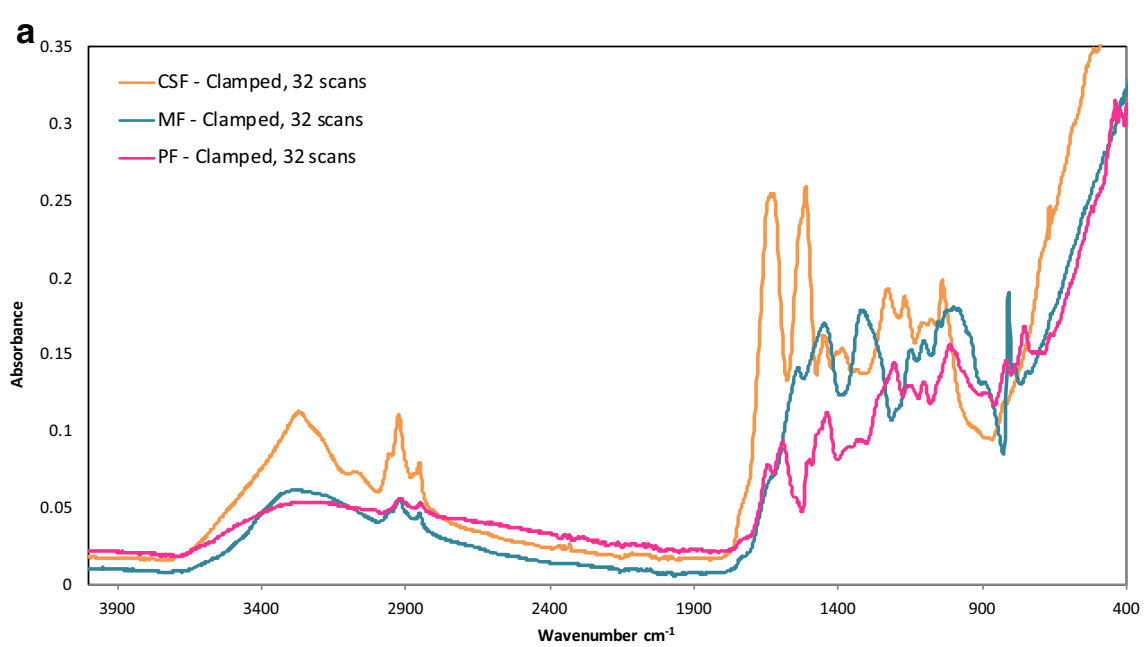

b
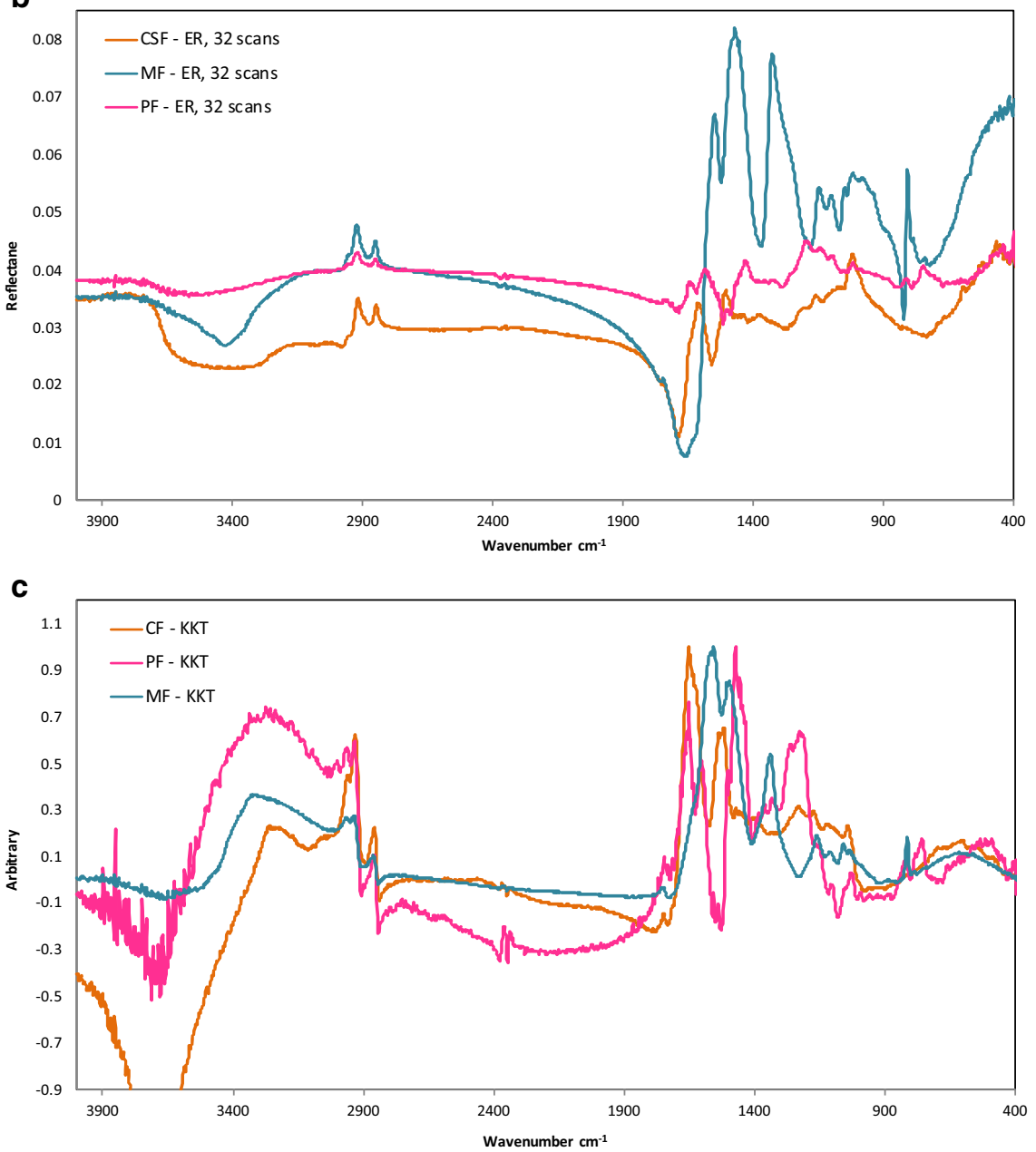

Fig. 9 Combined FTIR spectra of CF, PF, MF reference sheets with a ATR clamped, $\mathbf{b}$ ER sampling techniques, $\mathbf{c}$ KKT 
irregular or curved surfaces, the smaller aperture makes it easier for the camera to focus on the surface. It is possible to calculate absorbance spectra from the reflectance data via the KKT, however, band distortions and scattering can occur, resulting in bands of different shape and position being generated $[16,28]$. This can interfere with spectral matching and further complicates interpretation, so the KKT must be used with caution, and ideally by a conservation scientist.

Practical restrictions in the context of collection surveys, such as the time taken to conduct measurements, physical restrictions and personnel required are vital considerations. Conservator's professional duties are primarily driven by exhibition, acquisitions and loans, it is rarely possible to conduct retrospective surveys and analysis. When possible, discreet time periods and limited personnel are allocated with performance goals to be met. As such, survey activities, including polymer identification, need to be efficient and streamlined. 128 scans at $3 \mathrm{~min}$ per measurement, is unproductive resulting in a limited number of objects analysed in a given time. As this investigation demonstrated, the decrease in SNR achieved through cumulative scans produces little additional benefit above 32 scans.

When assessing the occupational, health and safety (OHS) considerations for FTIR analysis using the ATR manual handholding technique, 3 min for 128 scans is a potential physical risk. While the research objects chosen for this investigation were able to be clamped in contact with the ATR crystal, objects in collections must be handled with caution due to their value and significance. The unstable nature of historic plastic objects also means many will be too fragile to withstand pressure from the clamp. Consequently, a high number of collection items require analysis using the handheld technique, rather than clamping. As experienced, it can be challenging to hold objects in contact with the crystal consistently for long periods of time while acquiring measurements. Slight slips or changes in pressure result in inconsistent or low signal strength, low SNR values or rejected scans, further increasing time taken. Throughout this investigation, 32 scans, taking $40 \mathrm{~s}$, effectively balanced physical and professional consideration with achieving consistent pressure.

A flowchart for applying ATR and ER spectroscopy for the identification three-dimensional historic plastic objects during museum collection surveys has been developed (Fig. 10). It is intended to guide conservators in applying the appropriate module and contact technique to unknown polymers during collection surveys. Additional advice for each technique observed during this investigation are provided (Table 6).
A comparison was made of the availability of IR reference spectra obtained from commercial, published and in-house sources (Table 7). There are limitations with the reference spectral libraries as only one source provides spectra measured in reflectance mode, and no single source provides spectra for all polymers found in museum collections. To address this, a reference spectral library including at least 3 examples of each polymer type, in both absorbance and reflectance modes is required. Reference collections available commercially, published in literature, and sourced from plastics conservation workshops were also scrutinised (Table 7). As with the spectral libraries, no complete set is currently available. In addition, issues have been identified with incorrect labelling of samples in workshop sets and the commercially available ResinKit ${ }^{\mathrm{TM}}[19,36]$. Misidentified or mislabelled reference materials pose a risk of incorrect spectral interpretation and polymer identification. If a reference sample collection is to be developed in house, it requires replaceable, easily accessible sources for all polymers that can be cross-verified by a number of techniques.

\section{Conclusions}

This paper investigated a number of aspects of the FTIR instrument measurement process for identifying polymers in three-dimensional objects during museum collection surveys by conservators. It has been demonstrated that SNR is impacted by material type and surface characteristics far more than scan number, contact technique or aperture size. The ATR and ER modules are each more suitable for certain polymers and surface characteristics, and are optimised when used cooperatively. When the object necessitates, applying manual pressure to create contact with the ATR crystal is adequate to achieve a spectrum suitable for polymer identification. 32 co-added scans achieves a balance between maximising signal strength, minimising noise in the spectra and reducing OHS issues across polymer types, FTIR modules and contact techniques. Minimum SNRs to achieve identification vary by polymer type, and sampling technique, but in general need to be greater than $\sim 0.5$ for absorbance, and $\sim 5$ for reflectance. No single source of reference spectra or samples currently covers all polymers found in museums. Due to the limited availability of reference materials, material acquisition, material sharing, communication and collaboration across museums, conservation centres and universities is crucial to ensure the identification of polymers in museums can be conducted effectively. Conservators can use the developed workflow to apply ATR and ER FTIR for identification of polymeric materials during collection surveys. 


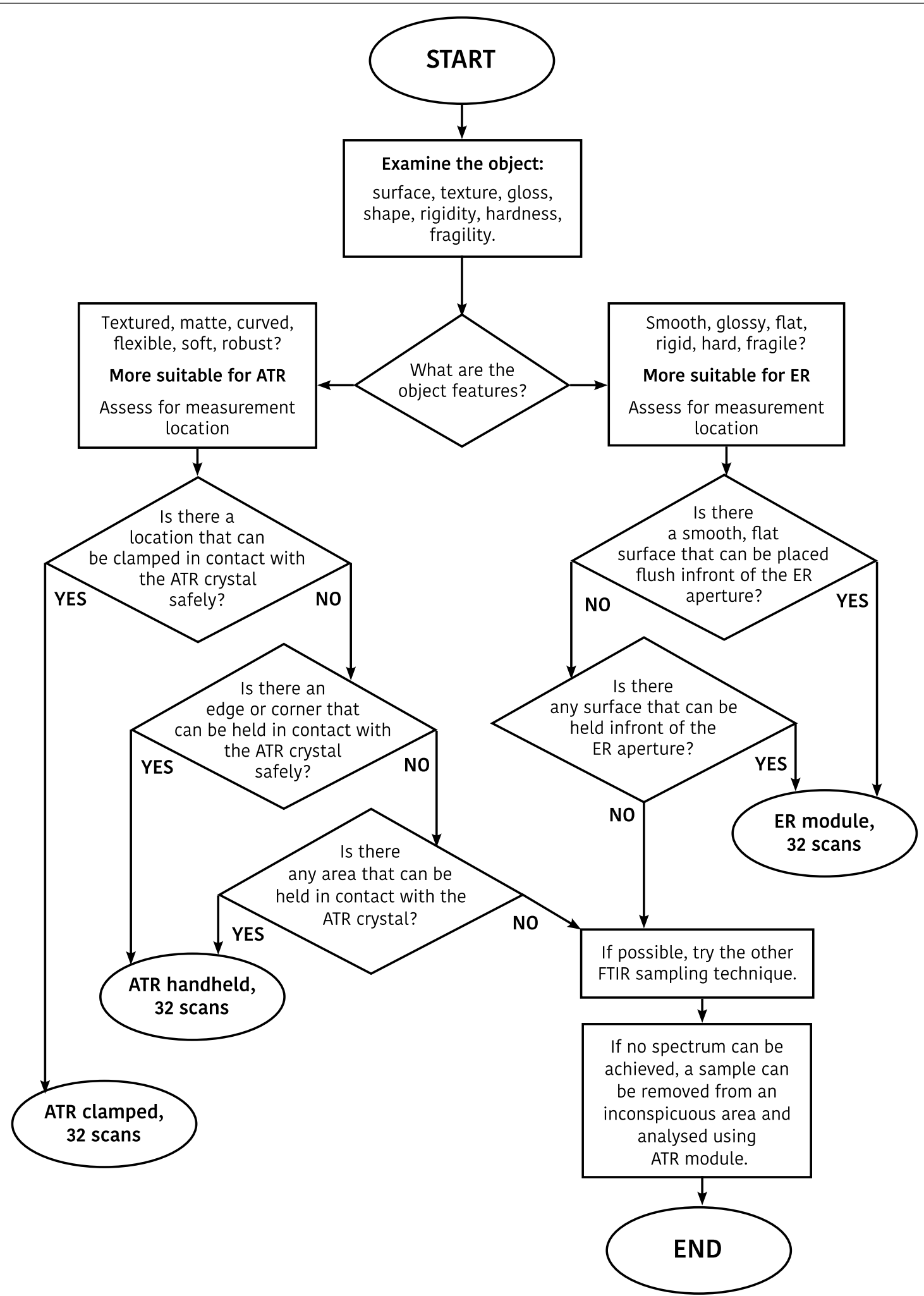

Fig. 10 Flowchart for applying ATR and ER FTIR identification to plastics in museum collections 
Table 6 FTIR measurement advice for three-dimensional polymeric objects

\begin{tabular}{|c|c|c|}
\hline ATR clamped & ATR handheld & ER \\
\hline $\begin{array}{l}\text { To make good contact with the ATR crystal }{ }^{\text {a }} \text {, } \\
\text { ensure flat or convex surface selected } \\
\text { Use a card barrier to project object surface from } \\
\text { being marked by the clamp }\end{array}$ & $\begin{array}{l}\text { To make good contact with the ATR crystal }{ }^{\mathrm{a}} \text {, } \\
\text { ensure: } \\
\text { - Consistent pressure applied to object (standing } \\
\text { upright over FTIR may assist) } \\
\text { - Sample does not shift (may be useful for } \\
\text { another person to operate the computer) } \\
\text { If unable to apply consistent pressure, select } \\
\text { lower number of co-added scans }\end{array}$ & $\begin{array}{l}\text { Select appropriate aperture size: } \\
\text { - Small for irregular or curved surfaces (present } \\
\text { convex surface) } \\
\text { - Medium or large if surface is flat } \\
\text { Support the object with blocks } \\
\text { Use the camera to ensure object is in focus } \\
\text { Do not shift or bump during measurement } \\
\text { If required, increase co-added scans to improve } \\
\text { spectral quality }\end{array}$ \\
\hline
\end{tabular}

a An initial pre-scan allows the operator to evaluate whether good contact has been achieved resulting in a good quality spectrum

Table 7 Plastic reference materials

\begin{tabular}{|c|c|c|c|}
\hline Source & Materials & FTIR module & Availability \\
\hline Infrared and Raman Users Group (IRUG) & 161 synthetic resins & Transmission, DRIFTS, ATR & Open source \\
\hline Bruker Polymer Library (2010) & 117 polymers & ATR & With instrument purchase \\
\hline Bruker Complete Library (2018) & 26,000 compounds & ATR & $\begin{array}{l}\text { \$7000 AUD with instru- } \\
\text { ment purchase }\end{array}$ \\
\hline Shashoua [32] & 15 historic polymers & ATR & Published book \\
\hline Saviello et al. [15] & 62 polymers & ATR, ER & Journal article appendix \\
\hline In-house reference spectra & 15 adhesives, 25 polymers & ATR, ER & - \\
\hline ResinKit $^{\mathrm{TM}}$ & 50 modern thermoplastics & - & $\sim \$ 400$ AUD \\
\hline Thea can Oosten workshop (2005) samples & 17 historic polymers & - & No longer available \\
\hline Yvonne Shashoua workshop (2015) samples & 16 historic polymers & - & No longer available \\
\hline POPART SamCo Kit & 46 plastic samples and objects & - & Available for loan \\
\hline
\end{tabular}

\section{Supplementary information}

Supplementary information accompanies this paper at https://doi. org/10.1186/s40494-019-0336-0.

Additional file 1. List of major peaks for all spectra including: polymer references sheets with ATR clamped and ER sampling techniques, case study objects with ATR clamped, handheld and ER sampling techniques, and KKT spectra.

\section{Abbreviations}

ABS: acrylonitrile butadiene styrene; ATR: attenuated total reflection; AICCM: Australian Institute for the Conservation of Cultural Material; CSF: casein formaldehyde; CA: cellulose acetate; CN: cellulose nitrate; ER: external reflectance; FTIR: Fourier transform infrared; GLAM: galleries, libraries, archives and museums; GRP: glass reinforced polyester; HQ: hit quality; IR: infrared; IRUG: infrared and Raman users group; KKT: Kramers-Kronig Transformation; MF: melamine formaldehyde; NR: natural rubber; OHS: occupational health and safety; P-P: peak-to-peak; PF: phenol formaldehyde; PA: polyamide; PBT: polybutylene terephthalate; PC: polycarbonate; PE: polyethylene; PET: polyethylene terephthalate; PETG: polyethylene terephthalate glycol; PMMA: poly(methyl methacrylate); PP: polypropylene; PS: polystyrene; PUR: polyurethane; PVC: poly(vinyl chloride); SNR: signal-to-noise ratio; SI: silicone; SBR: styrene butadiene rubber; VOC: volatile organic compound.

\section{Acknowledgements}

The authors acknowledge the contribution of Dr. Rosemary Goodall (Museums Victoria), Koman Tam (Bruker), Sharon Wong, Cancy Chu and Bronwyn Cosgrove.

\section{Authors' contributions}

JB and PN initiated the project. PN sourced reference objects, JB collected, analysed and interpreted FTIR data, under the supervision of PN. All authors read and approved the final manuscript.

\section{Funding}

This research is supported fully by the Australian Government through the Australian Research Council's Linkage Projects funding scheme (LP160100160).

Availability of data and materials

The datasets supporting the conclusions of this article are included within the article and its additional file.

\section{Competing interests}

The authors declare that they have no competing interests.

\section{Author details}

${ }^{1}$ Grimwade Centre, School of Historical and Philosophical Studies, University of Melbourne, Parkville, VIC 3010, Australia. ${ }^{2}$ Centre for Forensic Science, University of Technology Sydney, Ultimo, NSW 2007, Australia.

Received: 8 July 2019 Accepted: 5 November 2019

Published online: 18 November 2019

\section{References}

1. Casadio F, Toniolo L. The analysis of polychrome works of art: 40 years of infrared spectroscopic investigations. J Cult Herit. 2001;2:71-8.

2. Mitchell G, France F, Nordon A, Tang PL, Gibson LT. Assessment of historical polymers using attenuated total reflectance-Fourier transform infra-red spectroscopy with principal component analysis. Herit Sci. 2013;1(28):1-10. 
3. Nel P, Lonetti C, Lau D, Tam K, Sagona A, Sloggett R. Analysis of adhesives used on the Melbourne University Cypriot pottery collection using a portable FTIR-ATR analyser. Vib Spectros. 2010;53:64-70.

4. Keneghan B. Identification of plastics in cultural heritage collections by Fourier transform infrared spectroscopy (FTIR). Anal Methods. 2018;10:687-9.

5. Lavédrine B, Fournier A, Martin G. Preservation of Plastic ARTefacts in museum collections. Paris: Comit Des Travaux Historiques Et Scientifiques; 2012.

6. Tsang J-S, Madden O, Coughlin M, Maiorana A, Watson J, Little NC, Speakman RJ. Degradation of 'Lumarith' Cellulose Acetate. Stud Conserv. 2009;54(2):90-105.

7. Sustar V, Kolar J, Lusa L, Learner T, Schilling M, Rivenc R, Khanjian H, Kolesa D. Identification of historical polymers using near-infrared spectroscopy. Polym Deg Stab. 2014;107:341-7.

8. Schilling M, Learner T. Evolved gas analysis as a tool for characterizing plastics. In: Bridgland J, editor. ICOM-CC $16^{\text {th }}$ triennial conference Lisbon 19-23 September 2011: preprints. Lisbon: ICOM Committee for Conservation; 2011.

9. Pereira A, Candeias A, Cardoso A, Rodrigues D, Vandenabeele P, Caldeira A. Non-invasive methodology for the identification of plastic pieces in museum environment-a novel approach. Microchem J. 2016;124:846-55.

10. Curran K, Strlič M. Polymers and volatiles: using VOC analysis for the conservation of plastic and rubber objects. Stud Conserv. 2015;60(1):1-14.

11. Lattuati-Derieux A, Egasse C, Thao-Heu S, Balcar N, Barabant G, Lavédrine B. What do plastics emit? HS-SPME-GC/MS analyses of new standard plastics and plastic objects in museum collections. J Cult Herit. 2013;14:238-47.

12. Mitchell G, Higgitt C, Gibson LT. Emissions from polymeric materials: characterised by thermal desorption-gas chromatography. Polym Deg Stab. 2014;107:328-40.

13. Van Oosten T, Learner T. Identification and characterization of plastic artefacts: Which plastics are in my collection? The need for a plastic reference sample collection (SamCo). In: Lavédrine B, Fournier A, Martin G, editors. Preservation of Plastic ARTefacts in museum collections. Paris: Comit. Des Travaux Historiques Et Scientifiques; 2012. p. 37-42.

14. Chalmers JM. Infrared spectroscopy: sample presentation. In: Worsfold $P_{\text {, }}$ Townshend A, Poole C, editors. Encyclopedia of analytical science. 2nd ed. Oxford: Elsevier; 2005. p. 402-15.

15. Saviello D, Toniolo L, Goidanich S, Casadio F. Non-invasive identification of plastic materials in museum collections with portable FTIR reflectance spectroscopy: reference database and practical applications. Microchem J. 2016;124:868-77.

16. Picollo M, Bartolozzi G, Cucci C, Galeotti M, Marchiafava V, Pizzo B. Comparative study of Fourier transform infrared spectroscopy in transmission, attenuated total reflection, and total reflection modes for the analysis of plastics in the cultural heritage field. J Appl Spectrosc. 2014;68(4):389-97.

17. Cucci C, Bartolozzi G, Marchiafava V, Picollo M, Richardson E. Study of semi-synthetic plastic objects of historic interest using non-invasive total reflectance FT-IR. Microchem J. 2016;124:889-97.

18. Lebeaux R. The complete guide for identifying and testing plastic resins. Woonsocket: The ResinKit Company; 2013

19. Laganà A, Keneghan B. Which plastics are in my collection? The need for a plastic reference sample collection (SamCo). In: Lavédrine B, Fournier A, Martin G, editors. Preservation of Plastic ARTefacts in museum collections. Paris: Comit. Des Travaux Historiques Et Scientifiques; 2012. p. 37-42.
20. Smith GD, Kastenholz H, Chen V, Wilson A. Reassessing reference materials: surprises encountered when building a spectral library. In: Paper presented at the 13th Infrared \& Raman Users Group Conference; Sydney; 2018.

21. Sellors J, Smith P. FT-IR Identification: the expertise required to ensure compliance. Pharm Technol Eur. 2011;23:9.

22. Smith BC. How to properly compare spectra, and determining alkane chain length from infrared spectra. J Spectrosc. 2015;30(9):40-6.

23. Infrared and Raman Users Group. 2019. http://www.irug.org. Accessed 19 Feb 2019.

24. Hoffmann H, Leitner T. Surface analysis: infrared spectroscopy. In: Worsfold P, Townshend A, Poole C, editors. Encyclopedia of analytical science. 2nd ed. Oxford: Elsevier; 2005. p. 543-54.

25. Stuart BH. Analytical techniques in materials conservation. Chichester: Wiley; 2007.

26. Bell J, Palmer K, Smith A, Cannon A, McCarthy G, Nel P. The PolyMuse Online Heritage Resource Manager (OHRM): An australia-wide polymer database for the museum industry. In: Paper presented at American Institution for conservation meeting, New England; 2019.

27. Spragg R. Technical Note: Reflection measurements in IR spectroscopy. Seer Green: PerkinElmer; 2013

28. Derrick M, Stulik D, Landry JM. Scientific tools for conservation: infrared spectroscopy in conservation science. Los Angeles: The Getty Conservation Institute; 1999.

29. Baurecht D, Neuhäusser W, Fringeli UP. Modification of time-resolved step-scan and rapid-scan FTIR spectroscopy for modulation spectroscopy in the frequency range from $\mathrm{Hz}$ to $\mathrm{kHz}$. In: Fourier transform spectroscopy: 11th international conference proceedings; 1998; Athens, Georgia. American Institute of Physics. 1998; 430(1):367-70.

30. Blitz JP, Klarup DG. Signal-to-noise ratio, signal processing, and spectral information in the instrumental analysis laboratory. J Chem Educ. 2002;79(11):1358-60

31. OPUS/IR Reference Manual. Version 7. Bruker Optik GmbH: Ettlingen; 2014.

32. Shashoua Y. Conservation of plastics: materials science, degradation and preservation. Oxford: Butterworth-Heinemann; 2008.

33. Agilent Technologies. Highest available signal-to-noise performance, delivering superior sensitivity and analytical performance: technical overview. California: Agilent Technologies; 2011.

34. Spragg R. IR spectroscopy sample preparation methods. In: Lindon JC, Tranter GE, Koppenaal DW, editors. Encyclopedia of spectroscopy and spectrometry. 3rd ed. San Diego: Elsevier; 1999. p. 1210-7.

35. Fellgett PB. The multiplex advantage. Ph.D. Dissertation. Cambridge: University of Cambridge; 1951.

36. Eisenreich N, Rohe T. Infrared spectroscopy in analysis of plastics recycling. In: Meyers RA, editor. Encyclopedia of analytical chemistry: application, theory and instrumentation. New York: Wiley; 2000.

37. Lauridsen CB, Hansen LW, Brock-Nannestad T, Bendix J, Simonsen KP. A study of stearyl alcohol on Dan Hill PVC dolls and the influence of temperature. Stud conserve. 2017;62(8):445-55.

38. Coates J. Vibrational spectroscopy: instrumentation for infrared and Raman spectroscopy. Appl Spectrosc Rev. 1998;33(4):267-425.

\section{Publisher's Note}

Springer Nature remains neutral with regard to jurisdictional claims in published maps and institutional affiliations. 